\title{
Enseñar a ser médicos: un análisis de opinión de los médicos implicados en la docencia de la clínica práctica (II). Análisis cuantitativo de la opinión de médicos implicados en la docencia de clínica práctica
}

\author{
Jesús Millán Núñez-Cortés, José Antonio Gutiérrez-Fuentes
}

\begin{abstract}
Resumen. En un trabajo previo se elaboró, mediante un análisis cualitativo, una encuesta estructurada para conocer la opinión del profesorado implicado en la enseñanza de la clínica práctica. Dicha encuesta se ha empleado en una muestra significativa de 435 personas implicadas en la docencia en el entorno clínico, correspondiente a catedráticos de universidad, profesores titulares de universidad, profesores asociados de ciencias de la salud, personal asistencial sin vínculo docente y médicos internos residentes, pertenecientes a tres hospitales adscritos a la Universidad Complutense de Madrid. La valoración global de la enseñanza de la medicina es favorable en los dos tercios de los encuestados; no así la docencia práctica, que menos de un $50 \%$ la considera buena o muy buena. Se analizan los factores que son considerados tanto favorables como desfavorables por el profesorado en lo concerniente a la enseñanza práctica, y su opinión sobre la enseñanza de distintas áreas competenciales. Asimismo se valoran los factores considerados como elementos clave para la enseñanza práctica y para la correcta integración del alumno en los servicios clínicos, que se consideran un elemento limitante. Cabe señalar que el entorno que se considera más adecuado es la integración plena del alumno, con horario amplio, funciones concretas y estancias prolongadas en los distintos servicios.
\end{abstract}

Palabras clave. Opinión del alumnado. Opinión del profesorado. Práctica clínica.

Teaching how to be a doctor: an analysis of the opinions of doctors involved in the teaching of clinical practice (II). A quantitative analysis of the opinions of doctors involved in the teaching of clinical practice

Summary. In a previous study a qualitative analysis was used to produce a structured survey in order to find out the opinions of members of teaching staff involved in teaching clinical practice. This survey was applied to a significant sample of 435 people involved in teaching within the clinical setting. These respondents were university professors, tenured university lecturers, associate health science lecturers, healthcare personnel with no link to teaching and medical residents, all belonging to three hospitals affiliated to the Universidad Complutense of Madrid. Two thirds of those who answered the survey rated the teaching of medicine overall as favourable. This was not so, however, in the case of practical teaching, rated as good or very good by less than $50 \%$ of respondents. An analysis was performed of the factors that members of teaching staff see as favourable and unfavourable with regard to practical teaching, together with their opinion about the teaching of different areas of competence. Likewise, the factors that are considered key elements for practical teaching and for the successful integration of students into clinical services are also evaluated, since they are considered a limiting element. The setting that is considered to be the most appropriate is one in which the student is fully integrated, with a good number of hours, specific functions and long stays in the different services.

Key words. Clinical practice. Students' opinions. Teachers' opinions.

\section{Introducción}

En un trabajo previo se ponían de manifiesto los resultados de un análisis cualitativo sobre la enseñanza de la medicina en nuestro entorno [1]. Algunos de los resultados más significativos fueron:

- En los actuales planes de estudio, la función docente está poco valorada y el contenido teórico es excesivo y resulta con frecuencia irrelevante.
- La enseñanza está centrada en el 'profesor que informa' más que en el 'estudiante que aprende.'

- La metodología es poco activa y sigue teniendo como base la lección magistral (aunque, obviamente, no se pueda prescindir de ella).

- La docencia práctica ha aumentado pero no ha ganado en calidad (como consecuencia, en gran medida, de que las actividades prácticas son todavía muy pasivas y poco estructuradas).
Departamento de Medicina. Facultad de Medicina. Cátedra de Educación Médica Fundación Lilly-Universidad Complutense. Madrid, España.

Correspondencia: Prof. Jesús Millán Núñez-Cortés. Departamento de Medicina. Facultad de Medicina. Universidad Complutense. Ciudad Universitaria. E-28040 Madrid.

E-mail: jesus.millan@madrid.org

Conflicto de intereses: No declarado.

Competing interests: None declared.

(c) 2013 FEM 
Figura 1. Valoración de la enseñanza de la medicina.

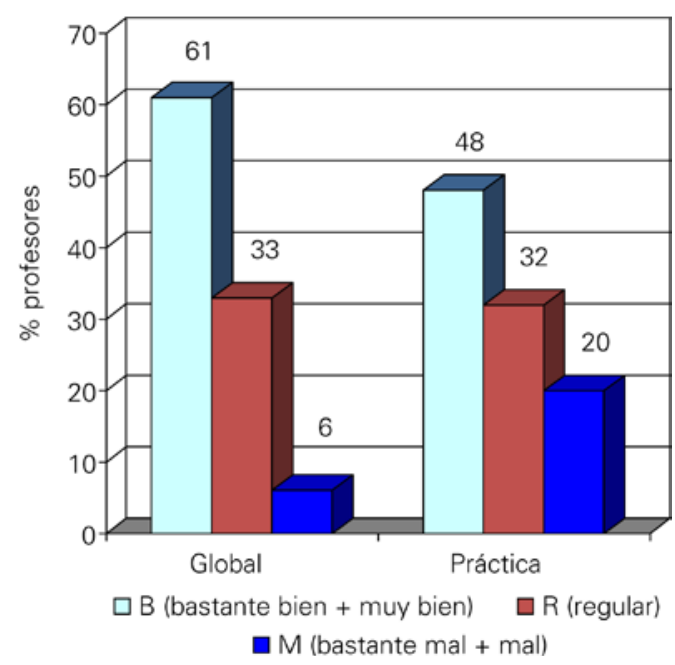

Figura 2. Valoración de los factores positivos por estamentos. CU: catedráticos de universidad; TU: titulares de universidad; ASCS: profesores asociados en ciencias de la salud; NOD: sin vínculo docente; MIR: médicos internos residentes.

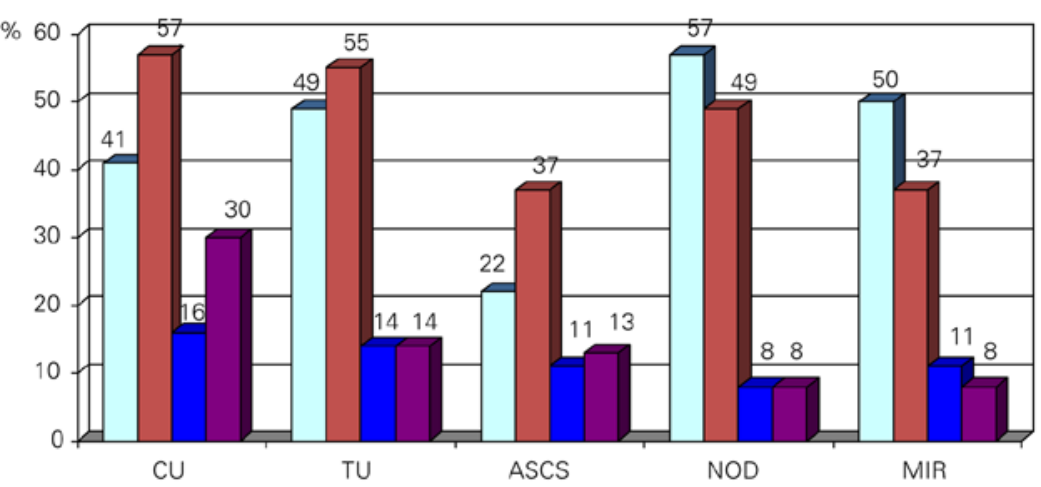

$\square$ Buena preparación y formación previas $\square$ Buena enseñanza teórica con programas completos - Cambios introducidos en la enseñanza
- Los métodos de evaluación se centran fundamentalmente en conocimientos teóricos.

- Entre el período preclínico y el clínico hay una alarmante falta de integración y la excesiva autonomía en la gestión departamental e individual de la docencia no ha resultado beneficiosa.

- El examen de médico interno residente (MIR) sigue presente en la actual formación médica de grado como referente formativo. No se cultiva la educación médica ni como formación ni como estructura de apoyo y hay una preocupante limitación de los recursos humanos y materiales para introducir cambios.

Este trabajo forma parte de un estudio más amplio, de corte sociológico, bajo la denominación de 'Enseñar a ser médicos', que se diseñó para conocer la opinión de los responsables de la enseñanza práctica de la medicina en los hospitales universitarios, para conocer sus opiniones, grado de satisfacción con el sistema actual de formación de médicos, carencias, deficiencias o disfunciones que perciben en él, y posibles propuestas o sugerencias para mejorarlo.

El objetivo prioritario es conocer la opinión de un grupo significativo de implicados en la enseñanza de la medicina práctica sobre la situación actual, al objeto de estar en condiciones de formular alternativas o mejoras en el entorno hospitalario, que es donde se produce mayoritariamente el proceso de enseñanza-aprendizaje de la práctica clínica.

\section{Materiales y métodos}

El estudio se llevó a cabo mediante entrevistas personales siguiendo un cuestionario previamente definido y estructurado, tal y como se definió en la parte cualitativa del estudio [1].

La aplicación del cuestionario se ha realizado con las siguientes características técnicas:

- Ámbito. Los tres hospitales universitarios vinculados durante el curso 2008-2009 a la Universidad Complutense de Madrid (UCM).

- Universo. Médicos con o sin condición docente formalmente reconocida, que asumen o tienen asignado algún tipo de docencia de práctica clínica de medicina en algunos de los tres hospitales.

- Tamaño y distribución de la muestra. El cuestionario fue realizado por un total de 435 profesores: 37 catedráticos (8\%), 51 profesores titulares (11\%), 196 profesores asociados (45\%), 74 profesionales médicos sin vínculo docente (17\%) y 77 MIR (17\%). Un 64\% de los profesores encuestados eran de sexo masculino, con un intervalo de edad predominante de 35-54 años (45\%). La distribución de los distintos grupos encuestados, estratificados por centros y categorías, se muestra en la tabla, donde se puede comprobar la representatividad de la muestra empleada.

Los resultados de los cuestionarios se trasladaron a una base de datos y se procesaron con el programa 
estadístico SPSS. Se analizaron las frecuencias de respuestas para cada apartado de forma global e individual y su asociación con el estamento docente encuestado: catedráticos, profesores titulares, profesores asociados, personal no docente y MIR.

\section{Resultados}

\section{Valoración de la enseñanza actual}

La valoración global de la enseñanza de la medicina fue considerada buena o muy buena por el $61 \%$ de los encuestados, mientras que la enseñanza de la práctica clínica sólo alcanzó este nivel para el $48 \%$ de los médicos entrevistados, siendo regular o mala para el otro $52 \%$. Por grupos docentes, los que mejor valoraron la enseñanza global de la medicina fueron catedráticos y profesores titulares $(68 \%$ y $71 \%$, respectivamente, la consideraban muy buena o buena). Aunque la enseñanza de la práctica clínica fue considerada mala o muy mala en porcentajes muy inferiores (27\% de los catedráticos, 26\% de los titulares y $35 \%$ de los profesores asociados), únicamente en el último caso el porcentaje de estos profesionales superó a aquellos cuya opinión fue positiva (30\%). Un resumen de estos resultados se muestra en la figura 1.

Se evaluaron de forma individual algunos aspectos positivos y negativos de la enseñanza actual, destacando que, para el total de los médicos entrevistados, los aspectos positivos más relevantes resultaban ser el alto nivel de los alumnos (51\%) y el alto nivel teórico y de los programas docentes (44\%), y como aspecto más negativo, la insuficiente enseñanza práctica (57\%). La opinión de los diferentes docentes en relación con los aspectos positivos no difiere de la opinión general, pero como aspecto negativo, el grupo de catedráticos y el de profesores titulares destacan el excesivo papel del examen MIR como referente (Figs. 2 y 3 ).

En lo referente a la enseñanza de las distintas áreas competenciales que incluían la valoración de las habilidades clínicas, los procedimientos diagnósticos y terapéuticos, el razonamiento y el juicio clínico, la comunicación, los valores profesionales y el desarrollo profesional, el conjunto de los docentes la consideraron buena o muy buena (70\%). Sin embargo, la valoración negativa superó a la positiva en el grupo de profesores asociados, que consideraron mala o muy mala la enseñanza de habilidades clínicas (37\%) frente a un $31 \%$ que la consideraron buena o muy buena, la comunicación (61\% frente a $21 \%)$, los valores profesionales (35\% frente a $34 \%$ ) y
Figura 3. Valoración de los factores negativos por estamentos. CU: catedráticos de universidad; TU: titulares de universidad; ASCS: profesores asociados en ciencias de la salud; NOD: sin vínculo docente; MIR: médicos internos residentes.

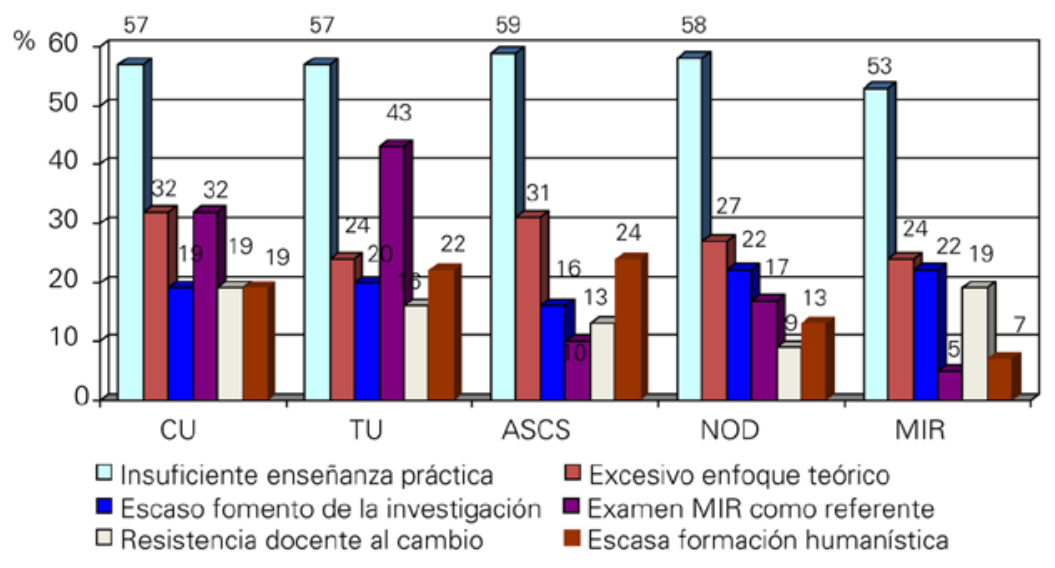

Tabla. Representatividad de la muestra estudiada $(n=4.950)$

\begin{tabular}{lccc}
\hline & $\begin{array}{c}\text { \% sobre colectivo total } \\
(n=4.950)\end{array}$ & $\begin{array}{c}\text { \% sobre } \\
\text { su colectivo }\end{array}$ & $\begin{array}{c}\text { \% sobre los encuestados } \\
(n=435)\end{array}$ \\
\hline Catedráticos & $1 \%$ & $84 \%$ & $8,5 \%$ \\
\hline Titulares & $1 \%$ & $75 \%$ & $11,7 \%$ \\
\hline Asociados & $12 \%$ & $33 \%$ & $45 \%$ \\
\hline No docentes & $56 \%$ & $3 \%$ & $17 \%$ \\
\hline Médicos residentes & $30 \%$ & $5 \%$ & $17,7 \%$ \\
\hline
\end{tabular}

el desarrollo profesional (37\% frente a $31 \%)$. Por otro lado, la valoración más positiva de cada área competencial de forma individual se obtuvo en los grupos de profesionales no docentes y de los MIR, superando en varios casos el 70\% (Fig. 4).

El aprovechamiento de los recursos docentes se evaluó en una escala de 0 a 10, considerándose los siguientes aspectos: materiales $(6,1)$, número de alumnos/profesor $(6)$, espacios $(5,9)$ y tiempo dedicado a la enseñanza $(5,8)$.

\section{Participación del profesorado}

La implicación docente de los distintos estamentos es superior para aquellos que tienen un vínculo docente y ejercen la docencia formal frente a aquellos 
Figura 4. Opinión sobre la enseñanza de distintas áreas competenciales. CU: catedráticos de universidad; TU: titulares de universidad; ASCS: profesores asociados en ciencias de la salud; NOD: sin vínculo docente; MIR: médicos internos residentes.

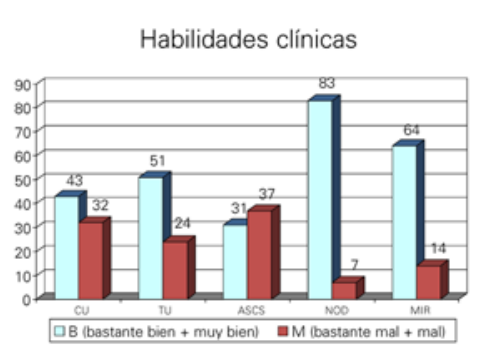

Comunicación

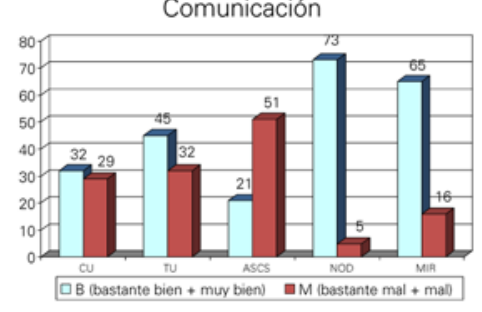

Procedimientos diagnósticos y terapéuticos

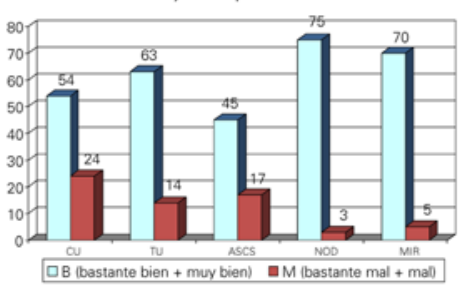

Valores profesionales

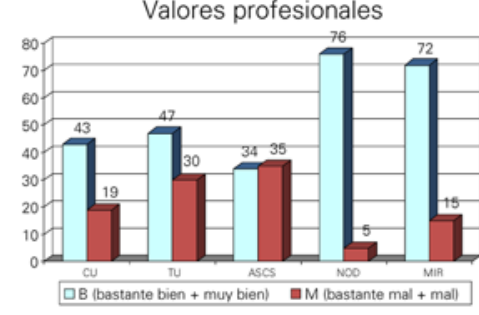

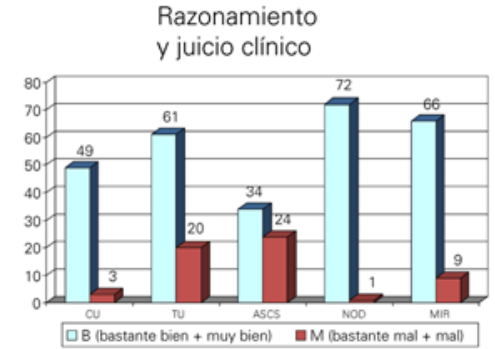

Desarrollo profesional

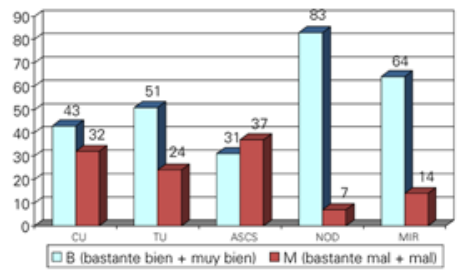

sin dicho vínculo (74\% frente a $61 \%)$. El $47 \%$ de los encuestados consideraron que todos los médicos deberían participar en la docencia. Destaca esta opinión en los estamentos de catedráticos (78\%), profesores titulares (71\%) y asociados (66\%) (Fig. 5). El $83 \%$ de todos los estamentos considera que la docencia debería ser voluntaria, siendo el grupo de los catedráticos el único que considera mayoritariamente que debería resultar obligatoria (51\%).

Los encuestados señalaban dedicar una media de 10,7 horas semanales a la docencia, de las cuales 9,8 horas se llevan a cabo al lado del alumno. Como resulta obvio, catedráticos y profesores titulares dedican más horas semanales de forma global a la docencia, de las cuales en una tercera parte están en contacto directo con los alumnos. Son los profesores asociados, el personal no docente y los MIR quienes dedican más horas de docencia 'al lado de los alumnos' en proporción con las horas totales (aproximadamente un 50\% de todas las horas dedicadas a la docencia).

\section{Participación del alumnado}

La opinión de los docentes sobre el nivel de preparación previa del alumno fue considerada adecuada y suficiente por el $57 \%$ del total, aunque el estamento de catedráticos la consideró algo peor (sólo el $54 \%$ de los catedráticos la consideraron adecuada).
Se solicitó la opinión de los encuestados sobre factores clave para la enseñanza práctica y qué era lo que consideraban más importante para el alumno. En ambos casos el factor más valorado fue la necesidad de un buen médico docente (Fig. 6).

La valoración más alta de los lugares idóneos para la práctica clínica fue, por este orden (en escala 0-10): áreas de hospitalización $(7,9)$, consultas $(7,4)$, urgencias $(7,2)$, quirófanos $(7)$, exploraciones especiales $(6,9)$ y centros de salud $(6,9)$.

Los encuestados consideraron que la formación práctica debía llevarse a cabo mediante la asistencia del alumno a sesiones clínicas (68\%), su integración en los diferentes servicios (58\%) y su rotación por menos servicios, permaneciendo en éstos más tiempo $(60 \%)$ en lugar de llevarse a cabo rotaciones breves en numerosos servicios (37\%).

Se analizó cuál debería ser la duración óptima de las prácticas en diferentes servicios. El conjunto de los estamentos docentes consideró que la rotación por los servicios de medicina interna y especialidades médicas debería ser superior (2-4 meses) a las rotaciones por los servicios quirúrgicos, médicoquirúrgicos, urgencias, cuidados intensivos y pediatría (1-2 meses). El mejor horario para la realización de las prácticas se consideró el de toda la mañana $(71 \%)$.

El 58\% de los encuestados se consideran favorables a la integración del alumno en los servicios clí- 
nicos, siendo este aspecto muy bien valorado por los profesores asociados (89\%). Los principales factores clave para la integración del alumno en los servicios clínicos fueron los indicados en la figura 7 .

\section{Discusión}

Del análisis de los datos cabe destacar en primer lugar la homogeneidad de las opiniones de los profesores de enseñanza de medicina de los tres hospitales vinculados a la UCM [2].

Este estudio pone de manifiesto que existe un campo para mejorar la opinión del profesorado sobre la enseñanza práctica de la medicina, el sistema actual de enseñanza y la adquisición de competencias clínicas.

Seis de cada diez médicos encuestados consideran adecuada la situación actual de la enseñanza de medicina; sin embargo, su valoración de la enseñanza práctica es inferior a la de la enseñanza en conjunto. Más de la mitad de los encuestados consideran que la enseñanza práctica es insuficiente (57\%) y que la enseñanza resulta excesivamente teórica $(27 \%)$.

Como es bien conocido, en el momento actual no siempre existe una adecuada correlación entre los métodos y los resultados educativos. Éstos se miden a través de evaluaciones cuyo resultado no siempre se correlaciona bien con el resultado educativo esperado, que debería centrarse en mejorar la atención al paciente $[3,4]$. Sin embargo, se mantiene una enseñanza excesivamente orientada a la consecución de óptimos resultados en el examen MIR, y ello hace que algunas áreas competenciales suelan ser deficitarias $[5,6]$.

La práctica totalidad de los profesores opina que los factores clave para una enseñanza práctica adecuada son la figura de un buen médico docente y la actitud e interés del alumno [7]. Además, los encuestados consideraron que la asistencia y participación del alumno en las actividades cotidianas de la práctica clínica, su integración en los diferentes servicios con la posibilidad de llevar a cabo actividades ante el paciente, y su rotación por un menor número de servicios con una mayor permanencia en ellos, es fundamental para lograr los objetivos marcados $[8,9]$. Distintos estudios previos han demostrado que el aprendizaje de la práctica clínica se encuentra directamente relacionado con el trabajo clínico cotidiano. La mayoría de las actividades de aprendizaje deberían relacionarse directamente con la atención al paciente, ya que la participación de los alumnos en su diagnóstico y tratamiento con-
Figura 5. Opinión sobre la necesidad de que todos los médicos deban de participar en la docencia práctica. CU: catedráticos de universidad; TU: titulares de universidad; ASCS: profesores asociados en ciencias de la salud; NOD: sin vínculo docente; MIR: médicos internos residentes.

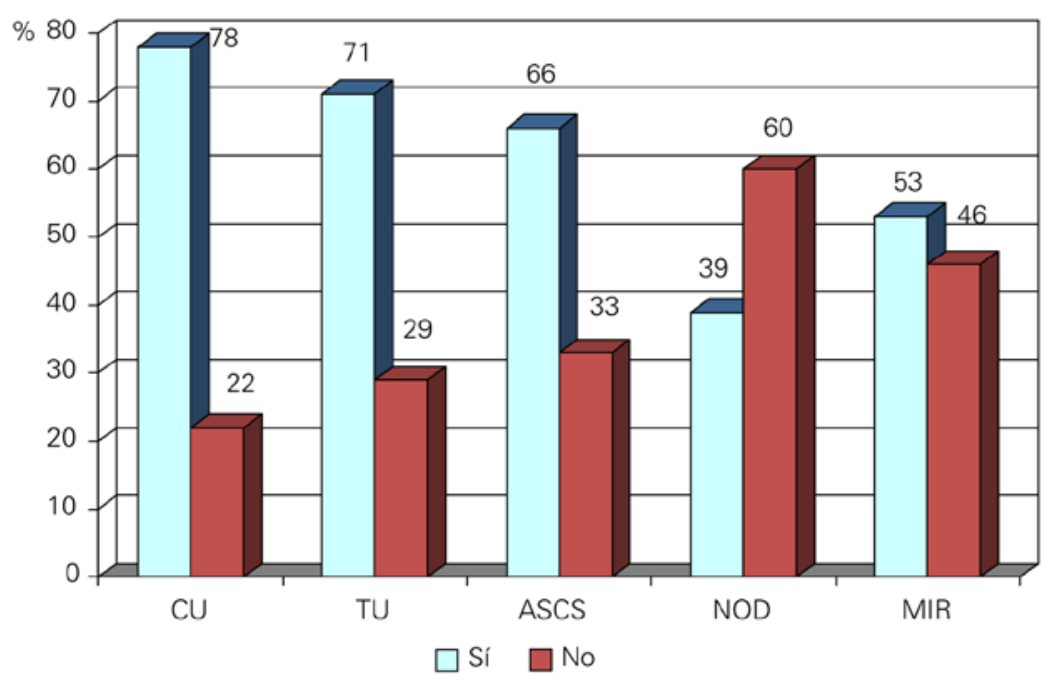

Figura 6. Factores considerados clave para la enseñanza práctica (escala 0-10).

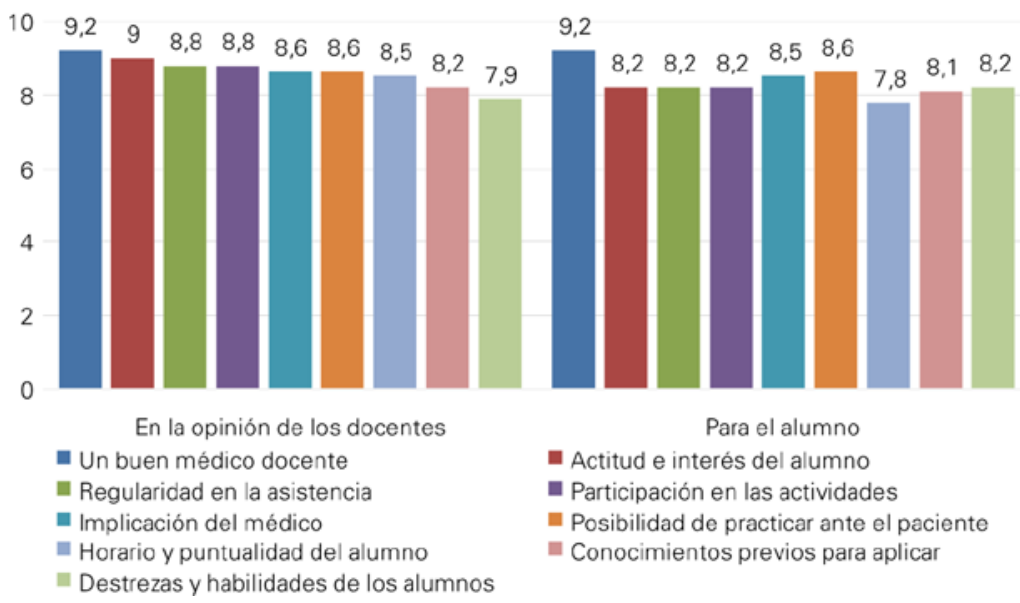

tribuye a la adquisición y el desarrollo de sus conocimientos $[10,11]$.

El $60 \%$ de los profesores se definen muy o bastante satisfechos con la labor docente que desempeñan, siendo esta proporción mucho mayor entre los profesores con reconocimiento formal docente, sobre todo entre los catedráticos y titulares. En con- 
Figura 7. Factores clave para una integración del alumno en los servicios clínicos.

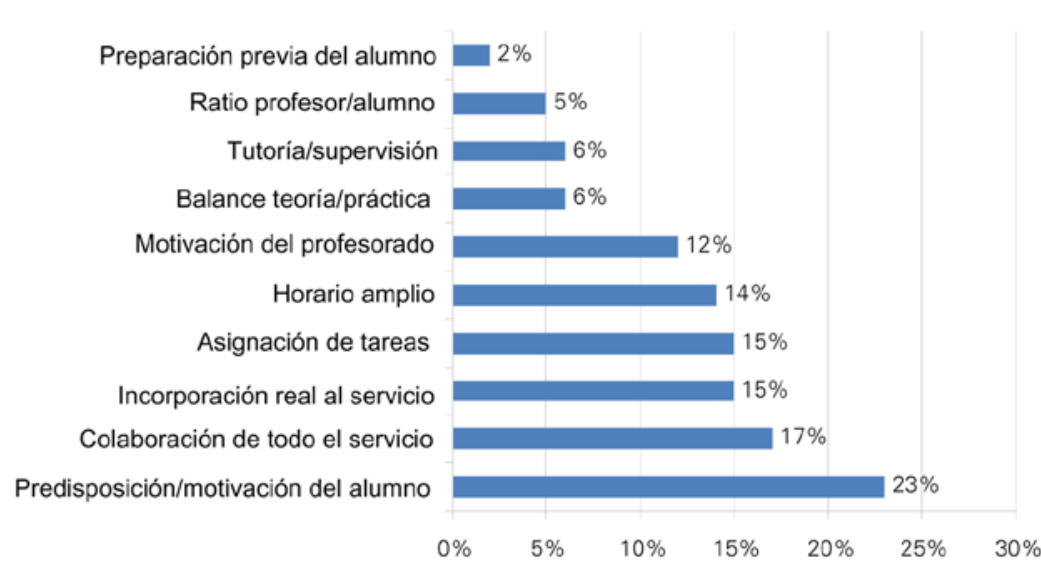

sonancia con esto, entre los profesores de medicina práctica se observa un mayor grado de implicación de aquellos que poseen un reconocimiento formal docente por parte de la universidad.

En el estudio que presentamos, la distribución de la muestra refleja la situación real y frecuente del profesorado en los hospitales universitarios, donde el profesorado asociado supera con creces en número a los profesores que son catedráticos y titulares, constituyendo éstos últimos únicamente un porcentaje minoritario del total de los docentes adscritos a los servicios clínicos. Este hecho resulta importante en la valoración de los resultados del estudio, por cuanto refleja la situación actual de la enseñanza de la medicina en el entorno clínico, en el que frecuentemente los profesores no funcionarios son críticos con el modelo de enseñanza, su participación puede estar mediatizada por otras tareas y su reconocimiento puede ser escaso. En la práctica, esto significa que se trata de un colectivo que por su número y función ha de tenerse muy en consideración.

En última instancia, los resultados obligan a considerar aquellas medidas que se pueden arbitrar para que todos aquellos que tienen la responsabilidad de transmitir los saberes médicos a los futuros médicos se sientan integrados y partícipes del sistema, redundando con ello en una mejoría de la calidad de la enseñanza.

Bibliografía

1. Millán Núñez-Cortés J, Gutiérrez-Fuentes JA. Enseñar a ser médicos: un análisis de opinión de los médicos implicados en la docencia de la clínica práctica (I). Conclusiones del análisis cualitativo y metodología para un estudio cuantitativo. Educ Med 2012; 15: 143-7.

2. Gutiérrez-Fuentes JA. La implicación de todo el profesorado: un elemento clave. Estudio 'Enseñar a ser médico'. Educ Med 2009; 12: 15-20.

3. Harden RM, Grant J, Buckley G, Hart IR. Best evidence medical education. Adv Health Sci Educ Theory Pract 2000; 5: 71-90.

4. Thistlethwaite J, Hammick M. The Best Evidence Medical Education (BEME) collaboration: into the next decade. Med Teach 2010; 32: 880-2.

5. Harden RM. The Objective Structured Clinical Examination (OSCE). In Hart I, Harden RM, eds. Further developments in assessing clinical competence. Montreal: Can-Heal Publications; 1987. p. 99-110.

6. Malone K, Supri S. A critical time for medical education: the perils of competence-based reform of the curriculum. Adv Health Sci Educ Theory Pract 2012; 17: 241-6.

7. Higgs J, McAllister L. Being a clinical educator. Adv Health Sci Educ Theory Pract 2007; 12: 187-200.

8. Millán Núñez-Cortés J. La enseñanza de las habilidades clínicas. Educ Med 2008; 11 (Supl 1): S21-7.

9. Díez-Lobato R, López-Encuentra A. La residentalización del alumno. Educ Med 2008; 11 (Supl 1): S43-5.

10. Van de Wiel MW, Van den Bossche P, Janssen S, Jossberger H. Exploring deliberate practice in medicine: how do physicians learn in the workplace? Adv Health Sci Educ Theory Pract 2011; 16: 81-95.

11. Michalec B. Learning to cure, but learning to care? Adv Health Sci Educ Theory Pract 2011; 16: 109-30. 